\title{
Table ronde : traduire Shakespeare aujourd'hui
}

Jean-Pierre Maquerlot

\section{(2) OpenEdition \\ Journals}

Édition électronique

URL : http://journals.openedition.org/shakespeare/1441

DOI : 10.4000/shakespeare. 1441

ISSN : 2271-6424

Éditeur

Société Française Shakespeare

Édition imprimée

Date de publication : 1 novembre 2000

Pagination : 275-293

ISBN : 2-84269-407-4

Référence électronique

Jean-Pierre Maquerlot, «Table ronde : traduire Shakespeare aujourd'hui », Actes des congrès de la Société française Shakespeare [En ligne], 18 | 2000, mis en ligne le 01 janvier 2007, consulté le 19 avril 2019. URL : http://journals.openedition.org/shakespeare/1441 ; DOI : 10.4000/shakespeare.1441 


\section{S H A K E S P E A R E \\ \& L A F R A N C E}

Société Française Shakespeare

Actes du Congrès de 2000

* * *

Textes réunis et présentés par

Patricia DORVAL

publiés sous la direction de Jean-Marie MAGUIN 
Colloque honoré d'une subvention du Ministère de l'Éducation Nationale, de la Recherche et de la Technologie

Site Internet $:<$ http://alor.univ-montp3.fr/SFS/> Liste de diffusion : <sfs-1@smr1.univ-montp3.fr>

Tous droits de traduction, de reproduction et d'adaptation réservés pour tous les pays.

(C) 2000. Société Française Shakespeare, École Normale Supérieure, 45 rue d'Ulm, 75005 Paris.

ISBN 2-84269-407-4 


\section{TRADUIRE S HAKE S PE A R E A U J O U R D' H U I}

Table ronde animée par Jean-Pierre Maquerlot avec la participation de Jean-Michel Déprats, Jean Malaplate, Sylvère Monod et Jean-Pierre Richard.

Permettez-moi tout d'abord de présenter brièvement nos invités même si leur notoriété rend la chose assez superflue.

- Jean-Michel Déprats dont les traductions sont très souvent jouées à Paris comme en province. Jean-Michel a traduit personnellement une bonne vingtaine de pièces sur les 37 ou 38 que Shakespeare a produites pour le théâtre. Il est le mâtre d'œuvre de la nouvelle édition des ouvres complètes à paraître chez Gallimard (la Pléiade). Il s'agira, cette fois, d'une édition bilingue; vaste projet collectif auquel travaille une équipe de spécialistes et traducteurs de Shakespeare placés sous sa direction.

- Jean Malaplate est le seul d'entre nous à avoir osé se mesurer aux Sonnets (éd. L'Âge d'Homme, puis Hachette «poche»); défi redoutable après les traductions de Henri Thomas, Pierre-Jean Jouve et Jean Fuzier, entre autres. Sa contribution au théâtre inclut Hamlet et Le roi Lear (Corti), Richard III (Bouquins). À paraître très prochainement: Peines d'amour perdues et Le songe d'une nuit d'été (Bouquins). Travail en cours : La Tempête.

- Sylvère Monod: admirable traducteur et «éditeur» de Dickens et de Conrad (la Pléiade); on lui doit une traduction de 
Henry $V$ (Club Français du Livre) republiée avec quelques infimes modifications chez Flammarion; un Beaucoup de bruit pour rien à paraître cette année (Bouquins). Sylvère achève présentement un Mesure pour mesure (traduction et appareil critique) dont la sortie (également chez Bouquins) est prévue courant 2001 ou 2002.

- Jean-Pierre Richard, traducteur justement célèbre dans le domaine du roman afro-américain et sud-africain contemporain, puis universitaire, est l'auteur d'un Beaucoup de bruit pour rien créé au théâtre de Lisieux au printemps 1999. Ses traductions de Titus Andromicus, Les deux nobles cousins et Les joyeuses commères de Windsor (en collaboration avec J.-M. Déprats) seront publiées en Pléiade.

- «Last but also least», votre serviteur, Jean-Pierre Maquerlot, recruté dans l' «équipe Pléiade» pour une traduction - à présent achevée - de Troïlus et Cressida et plusieurs introductions avec appareil critique (Henry $V$, La mégère apprivoisée, Tout est bien qui finit bien et Mesure pour mesure). J'ai également commis - non sans souffrances - une traduction du livre philosophicoshakespearien de Stanley Cavell, Disowning Knowledge, parue sous le titre Le déni de savoir (Le Seuil, 1993).

M'étant acquitté de cette tâche bien agréable et avant de suggérer certains axes qui pourraient structurer notre débat, je saisis l'occasion de cette tribune pour réaffirmer la dignité trop peu reconnue du métier de traducteur. Si chacun admet l'utilité sociale et culturelle de la traduction qui met à la portée d'un large public des textes autrement inaccessibles, on hésite encore trop à reconnaître que le traducteur est un artiste du langage. Ricœur dit avec justesse qu'écrire c'est toujours réécrire. La traduction atteste la vérité de la proposition réciproque : traduire c'est réécrire mais c'est aussi écrire. Restituer une parole travaillée - qu'elle soit dramatique ou, plus généralement, «littéraire» - quand bien même cette parole n'afficherait pas son caractère ouvertement «poétique», suppose un acte d'écriture homologue de celui qui l'a initialement produite. Rares sont les traducteurs qui se posent comme écrivains car ils se veulent «au service» d'un texte déjà constitué. Cependant, questionnés sur leur pratique, tous conviennent que la traduction est affaire d'inspiration, de sensibilité aux possibilités expressives des deux langues et de travail sur le langage qui est leur instrument; tous conviennent aussi que leur activité de décodage et de transcodage fait d'eux des interprètes en qui se conjuguent 
interprétariat (ou truchement) et interprétation, au sens que les comédiens, les musiciens ou les danseurs donnent à ce mot. Il faut avoir, me semble-t-il, un tempérament sinon un talent d'écrivain pour s'accommoder de cette liberté conditionnelle (ou surveillée) allouée au traducteur, quand les «gênes exquises» qui le cernent l'obligent à faire de nécessité vertu dans l'ordre de l'écrit.

Mais comme tout interprète, le traducteur se forge une politique, une philosophie qui guide sa pratique même s'il transgresse ponctuellement les principes qui la fondent. Trois types de problèmes viennent à l'esprit de quiconque réfléchit à la traduction théâtrale :

1) La question de la théâtralité du texte d'arrivée. Sachant que le texte initial - ici shakespearien - a été élaboré en fonction de sa destination scénique, comment restituer en français cette dimension constitutive de l'original ? Mais à donner la priorité absolue à l'oralisable, ne risque-t-on pas de dévaloriser ou d'occulter certaines significations saisissables par un public élisabéthain mais qui, aujourd'hui, passeraient mieux dans une traduction conçue pour la lecture?

2) L'historicité de la langue d'arrivée. Compte tenu de la distance temporelle qui nous sépare de ces textes, faut-il suggérer l'ancien ? Cela fait-il partie de notre devoir de fidélité ? Ne risque-ton pas alors de décevoir l'attente d'un public qui, légitimement, peut souhaiter se sentir de plain-pied avec la langue du spectacle, comme si Shakespeare était notre contemporain ?

3) La poétique du texte d'arrivée. Si la vertu poétique d'un texte ne se réduit pas à sa forme versifiée, il reste que le vers qui agence les mots selon une mesure, une durée, une cadence, contribue à l'effet poétique, effet esthétique mais aussi effet de sens. Mais alors quel vers choisir? L'alexandrin (rimé ou non) ou le vers libre? La prose n'est-elle pas mieux à même de concilier les exigences du rythme, de l'expressivité poétique et de la fluidité relative du vers blanc tel que Shakespeare le pratique dans ses œuvres de la maturité ?

$\mathrm{Si}$ vous êtes d'accord avec cette problématique, je propose que nous commencions par cette dernière question.

\section{Traduire Shakespeare en vers ? Si oui, quel vers ?}

Jean Malaplate, vous êtes connu comme un défenseur fervent et talentueux de l'alexandrin comme étant le médium le plus approprié pour rendre le vers blanc shakespearien. Pouvez-vous 
justifier ce choix ?

Jean MALAPLATE. Faut-il traduire les vers en vers? La question se pose pour l'ensemble de la poésie, depuis des siècles et chacun y apporte ses conceptions personnelles. Il en va de même pour le théâtre, y compris celui de Shakespeare. Voltaire, introducteur de notre dramaturge en France, répond d'emblée, le premier avant beaucoup d'autres, par l'affirmative. Il donne l'exemple en traduisant le monologue d'Hamlet en vers rimés et en déclarant: «Malheur aux faiseurs de traductions littérales qui en traduisant chaque parole énervent le sens. C'est bien là que la lettre tue et que l'esprit vivifie». On ne saurait mieux dire. Cependant la traduction voltairienne est si loin du texte, elle mêle à tel point les idées personnelles de Voltaire à l'original anglais qu'elle n'est guère un bon exemple à l'appui de sa thèse. Celle-ci, à mon avis, demeure entièrement valide à la lumière de l'expérience de deux siècles et demi et malgré le passage du temps. Une traduction en prose d'un texte en vers, même d'un texte théâtral, fait perdre sans recours à son modèle le rang d'objet esthétique, n'est plus qu'une information sur le contenu de l'original, mais non l'équivalent de celui-ci.

D'aucuns allèguent que le théâtre en vers est démodé, n'intéresse plus personne, que les poètes même - ou ceux qui s'affirment tels - s'expriment en prose aujourd'hui, qu'il faut moderniser le texte pour qu'il reste lisible et jouable. Mais on aurait alors un Shakespeare démodé en anglais et c'est la version française qui deviendrait la meilleure. Ne faudrait-il pas, selon la même logique, faire subir à Racine, Corneille, Hugo, Baudelaire, etc. la même dégradation? Le problème n'est pas de savoir comment on écrirait de nos jours, mais de respecter l'esthétique d'une époque qui est du reste partie intégrante de l'œuvre et à laquelle on doit la même fidélité qu'au sens nu.

Jean-Pierre MAQUERLOT. Soit. Mais alors quel vers ?

Jean MALAPLATE. Quel vers ? L'alexandrin, bien sûr, pour traduire le pentamètre iambique anglais, à la fois parce qu'il en est l'homologue dans notre littérature et parce que la plus grande brièveté de l'anglais l'exige pour le respect du sens. L'alexandrin blanc mais fortement rythmé pour le vers blanc anglais et le vers rimé comme correspondant du vers rimé anglais, même si l'on peut (et doit) renoncer parfois à certaines des exigences désuètes de notre versification classique : hiatus, diérèse, rimes masculines et féminines, etc. Mais ces irrégularités doivent demeurer dans un cadre cohérent, conserver une certaine rigueur métrique et une 
qualité formelle qui ne soit pas trop indigne de l'auteur traduit. Des vers boiteux, dépassant de plusieurs syllabes le rythme général qui a été adopté, ne peuvent que ruiner l'entreprise. De même, quel sens conserverait tel passage où l'acteur annonce qu'il va lire un sonnet si ce qui suit n'a rien d'un sonnet? Dans tel passage de Peines d'amour perdues où les personnages se renvoient des répliques triplement rimées et où l'un d'eux poursuit avec ironie par un vers absurde, comment répondrait-il, dans une traduction en prose, que ce qu'il dit est peut-être «- dépourvu de raison! - Mais bien pourvu de rime!».

Sans doute la traduction en vers, surtout rimés, est-elle beaucoup plus difficile que la traduction en prose, surtout si l'on s'impose de serrer d'aussi près que possible le sens de l'original. Elle ne peut être utilement pratiquée que par de bons versificateurs, sinon de bons poètes. Une honnête traduction en prose peut-être préférable à une mauvaise traduction en vers. Mais la contrainte est facteur de recherche plus approfondie et, par là, d'imperfection moins grande, de meilleur service rendu à un artiste de premier plan et à son lecteur.

Jean-Pierre MAQUERLOT. Il vous est arrivé, Jean-Michel Déprats, d'utiliser des alexandrins dans vos traductions, mais cela reste exceptionnel. Est-ce à dire que vous ne partagez pas le point de vue qu'on vient d'entendre?

Jean-Michel DÉPRATS. La poésie dramatique anglaise est une poésie accentuelle et rythmique et non quantitative ou syllabique. C'est dire que ni le décasyllabe ni l'alexandrin ne sont en français l'équivalent prosodique du pentamètre iambique shakespearien. L'alexandrin classique en est tout au plus l'équivalent culturel. Cadencé une fois pour toutes par la succession d'unités hexasyllabiques et la symétrie des hémistiches, il est d'une facture bien éloignée du vers dramatique élisabéthain, et l'accumulation d'alexandrins trouvant leur partage unique à la césure trahit la langue shakespearienne inégale, mouvante, et tourmentée. Traduire des vers comme «Blow, winds and crack your cheeks! Rage, blow !» (King Lear, III.2.1) ou «Inch-thick, knee-deep ; o'er head and ears a fork'd one. / Go, play, boy, play; thy mother plays, and I / Play too» (The Winter's Tale, I.2.186-8) par des alexandrins, c'est en trahir la mobilité, la pensée et la vie. En revanche, dans les premières comédies (Love's Labour's Lost, A Midsummer Night's Dream...), pour les envolées lyriques ou les tirades argumentatives, là où règnent la régularité rythmique, la rime, la rhétorique et la 
convention, l'alexandrin rimé est le bienvenu... et vient d'ailleurs naturellement sous la plume. Mais traduire tous les pentamètres iambiques dans toutes les pièces par des décasyllabes ou des alexandrins, c'est imposer un carcan étouffant et artificiel, tailler dans le sens et dégrader la forme en pur formalisme. La matière sémantique est trop riche pour pouvoir être engoncée dans le cadre rigide d'un vers régulier. Comme le note avec perspicacité Yves Bonnefoy (Études Anglaises $n^{\circ} 4$, oct-déc. 1964) dans un article où il analyse «les risques des formes fixes» en poésie, «la forme n'est pas un cadre mais un instrument de recherche [...]. Il n'y a pas de signification qui préexiste au poème, il n'y a qu'un destin qui a pris forme. Voilà pourquoi le vers régulier, dans la traduction, donne presque toujours une impression de gratuité, et parfois même de parodie». Versifier n'est pas écrire des vers et c'est une tâche beaucoup plus aisée et moins méritoire qu'on ne l'imagine. Traduire 30 pentamètres par 40 décasyllabes, c'est simplement faire tomber la toise à 10 , retourner à la ligne au bout d'une certaine «quantité» syllabique, dénaturer l'original en l'allongeant, alors que tout l'enjeu est d'être aussi concis. En conséquence, c'est la voie d'une traduction juxtalinéaire, privilégiant le rythme plutôt que le mètre, sans «clipping» ni «padding», sans couper dans la matière sémantique ni (plus rarement) étoffer, qui me paraît le mieux répondre aux sollicitations de l'original.

Jean-Pierre MAQUERLOT. Jean-Pierre Richard, votre activité de traducteur professionnel vous a confronté à la prose plutôt qu'aux vers. Comment réagissez-vous aux problèmes que pose la traduction en français d'un texte de théâtre écrit en vers blancs ?

Jean-Pierre RICHARD. À cet égard, je distinguerai deux domaines d'expérience, l'écrit et l'oral.

S'agissant de l'écrit, à mon sens la question n'est pertinente que si l'on peut nommer «vers» un dispositif d'ordre principalement graphique, un espace textuel marqué sur la page par un fréquent retour à la ligne. Le texte imprimé se trouve ainsi découpé en séquences d'une longueur - ou d'une brièveté ! - comparable, toutes bornées à gauche par une majuscule mais non systématiquement bornées à droite par une ponctuation forte. Ce code typographique, du seul fait qu'il rompt avec celui qui régit la prose, suffirait à constituer, au regard du lecteur, une versification, sans qu'intervienne en plus un mètre identifiable ou un jeu de rime systématique. 
On n'en perçoit pas moins immédiatement la différence avec le fonctionnement de l'original: dans les pièces de Shakespeare il n'est de "vers» sans un support métrique, à base d'iambes et autres pieds, attestés, reconnaissables, systématiquement mis en œuvre. Une traduction française en «vers typographiques», sans formule métrique ou phonique systématique, ne serait-elle pas, presque littéralement, de la poudre aux yeux ? Et si ce type de vers peut effectivement produire un effet sur le lecteur et le mettre à peu de frais en état de poésie pour ainsi dire, il échappera totalement au spectateur, qui reste par définition aveugle à la typographie... Sauf que ce parti pris, exclusivement graphique en apparence, a bel et bien une dimension rythmique. Par rapport au vers français qui me sert à traduire le vers shakespearien, je dirais même qu'elle n'est pas moins fondamentale ou constitutive que l'aspect typographique.

Quelle est la raison d'être du vers dans le texte original ? La métrique, à laquelle s'ajoute parfois la rime, permet à Shakespeare de créer une dynamique forte, en travaillant à l'échelle du vers une myriade de petites unités textuelles. Pour organiser son matériau verbal, l'auteur ne compte plus seulement sur la syntaxe et sur le jeu de contraintes et de possibilités d'ordre purement linguistique qu'elle autorise, mais aussi sur des procédés formels d'ordre stylistique, tels que le mètre, la rime, l'anaphore, l'assonance, l'enjambement, etc. La force du langage utilisé s'en trouve démultipliée.

Plusieurs éditions de Titus Andronicus font s'ouvrir l'Acte II sur ce monologue d'Aaron :

Now climbeth Tamora Olympus' top,

Safe out of fortune's shot, and sits aloft,

Secure of thunder's crack or lightning flash,

Advanced above pale envy's threatening reach.

Même si ces vers ne riment pas, ils jouent intensément du signifiant phonique, y compris d'une assonance («top» / «aloft») renforcée d'un écho intérieur au deuxième vers («shot»). Rythmes et sonorités ont constitué en anglais un jeu de forces dont il me faut essayer de créer une équivalence en français. Or, je ne pars absolument pas de zéro: Shakespeare m'a mâché le travail, lui qui a su se servir du vers anglais pour produire ces puissants effets. En sorte qu'il me suffit quasiment d'épouser le mouvement de son vers afin d'obtenir 
en français un effet comparable. Et seulement comparable, compte tenu bien entendu du taux regrettable de déperdition quand on passe d'une langue à l'autre. Mais, alors que le français et l'anglais ont une interface relativement étendue, plus que le swahili et le français par exemple, pourquoi irai-je surperposer au schéma rythmique shakespearien un mètre français - décasyllabe, alexandrin ou autre - dont la logique n'a rien à voir avec le texte à traduire ?

Jean-Pierre MAQUERLOT. Et pourtant vos traductions se présentent bel et bien comme une suite de tirades ou de répliques écrites selon une disposition qui rappelle les vers, même si ce sont des vers libres. S'agissant de l'extrait du monologue d'Aaron que vous venez de citer, on serait curieux de connaître la traduction que vous en avez faite.

Jean-Pierre RICHARD. Traduire le vers anglais par un vers français... Malgré le caractère évident de la proposition, est-ce bien la formule idéale de la fidélité ? Si, sans rime, sans mètre, je crois pouvoir mieux restituer l'énergétique du vers shakespearien, pourquoi m'embarrasser d'une contrainte extérieure à l'original, qui, au lieu de me servir, me compliquera la tâche, m'éloignera du but ? Le premier vers d'Aaron a un schéma ascendant qui culmine avec «top», que l'existence d'un génitif en anglais permet à Shakespeare de hisser iconiquement jusqu'en fin de vers. Certes, même si le français est dépourvu de cas génitif, il autorise l'inversion, mais une formulation du type «Tamora qui gravit de l'Olympe le sommet» pourra paraître, surtout à l'oreille du spectateur, bien alambiquée, ce que n'est jamais le langage d'Aaron dans cette pièce. Il m'a semblé préférable de traduire la dynamique, sans m'imposer d'autres contraintes - déjà bien assez lourdes et frustrantes - que celles liées au fonctionnement grammatical de ma langue d'arrivée:

Voici Tamora qui gravit l'Olympe jusqu'au sommet, Hors d'atteinte du sort, et qui s'asseoit là-haut, À l'abri du tonnerre ou de l'éclair, Loin de la jalousie blême.

Je ne vois pas ce que l'alexandrin ajouterait au dynamisme de l'original. Mieux : je ne vois pas ce que l'alexandrin de mon deuxième vers ajoute à la configuration dynamique créée par l'anaphore des attributs (+ préposition) placés en position initiale, tel que l'anglais la développe sur trois vers : «Safe (out of)» / «Secure (of)» / «Advanced (above)». Seule ici m'importait la restitution en français du trope de la relance rythmique et mon 
alexandrin n'est qu'un épiphénomène.

À la différence de l'alexandrin français et de tous ces autres mètres qui font violence à notre souffle, au débit «naturel» du français, le vers des pièces shakespeariennes ne correspond pas en général à un carcan métrique que l'auteur aurait imposé à un matériau verbal ; il paraît autant constitué par le rythme «naturel» d'un discours en anglais qu'il n'impose lui-même tel ou tel effet rythmique. La question du vers se pose donc doublement: interrogeons-nous sur ce que le rythme doit au vers, et autant, sinon plus, sur ce que le vers, en tant qu'unité métrique, doit lui-même au rythme de la langue, sur sa fonction ancillaire, sur son rôle de fairevaloir. Et tâchons seulement de traduire, non pas le vers shakespearien par le vers français, mais le rythme par le rythme.

Jean-Pierre MAQUERLOT. Sylvère Monod, vous êtes ici le seul à avoir traduit du Shakespeare sous l'œil vigilant d'un poète, Pierre Leyris ; or votre Henry $V$ est curieusement en prose !

Sylvère MONOD. Une précision d'abord sur mes coordonnées et mon itinéraire de traducteur shakespearien car effectivement ils conditionnent ou expliquent mes réponses aux questions posées : j'ai effectivement travaillé sous la férule de Pierre Leyris en traduisant Henry $V$ pour le Club Français du Livre au milieu des années 50 ; je collabore actuellement, sous l'égide du duo dijonnais MG/GM (Michel Grivelet et Gilles Monsarrat) au Shakespeare de «Bouquins»; j'y ai la charge de Much Ado et de Measure for Measure. Ma carrière est donc courte mais ses deux phases sont séparées par quelque 45 ans. Je tiens à souligner que dans les deux phases il s'agit d'éditions bilingues, car cela me paraît imposer au traducteur certaines contraintes: le texte et la traduction se font face et s'interpellent; dans «Bouquins», de surcroît, les notes sont en bas de page et ne peuvent manquer de souligner la démarche qui aura conduit de l'un à l'autre. Cela posé, venons à votre question : faut-il traduire les vers de Shakespeare en prose ou en vers français? Leyris m'avait laissé le choix entre prose et vers libre pour Henry $V$; je lui avais soumis un échantillon traité des deux manières et il avait estimé qu'il n'y avait guère de différence entre ma prose et mes vers ; mon Henry $V$ a donc été publié sous la forme de prose.

Jean-Pierre MAQUerLot. Ce qui ne vous empêchera pas, plus tard, d'opter pour le vers dans vos traductions pour «Bouquins». Est-ce une conversion? 
Sylvère MONOD. Je suis entré tardivement dans l'équipe de «Bouquins» pour répondre par amitié à un appel de détresse, car le succès des premiers volumes de l'édition imposait le recrutement rapide de nouveaux collaborateurs. Devenu membre de cette équipe, j'ai découvert qu'elle avait établi, au terme d'une authentique concertation, des règles, et même un «protocole» abondant et précis, qui m'a été d'un grand secours. Nous sommes invités à pratiquer ce qu'on pourrait appeler l'alexandrin assoupli, c'est-à-dire un alexandrin où les $e$ muets ne comptent pas et où on ne s'interdit pas de petites variations (une ou deux syllabes en plus de temps en temps); on est invité à pratiquer la rime chaque fois que Shakespeare l'a introduite (fins de scènes, chansons). Faute d'être un poète, je suis un homme de bonne volonté et j'ai été un versificateur assez abondant; j'ai donc pu me plier à cette discipline et y trouver un certain bonheur. Aujourd'hui je suis presque choqué quand je vois dans une édition bilingue des pavés de prose face aux vers de Shakespeare ; cela me donne une impression de platitude ou d'aplatissement - je ne veux pas dire que le vers du traducteur échappe toujours à la platitude (certains volumes de la "Collection Shakespeare» en fourniraient des exemples). Mais j'ai constaté que toute contrainte a ses vertus - que ce soit celle du sonnet, du limerick, du haiku - et que les obligations imposées pour «Bouquins» ont en tout cas le mérite de stimuler à la recherche de la densité, éminemment nécessaire à tout traducteur d'anglais en français, en particulier quand il s'agit de Shakespeare.

\section{Traduire Shakespeare dans une langue plutôt archaïsante, plutôt moderne ou résolument contemporaine ?}

Question rhétorique, direz-vous, puisqu'aucun des traducteurs ici présents (y compris moi-même) n'opte pour une langue uniformément archaïsante. Le recours à l'archaïsme est toujours sporadique et discret. Aussi, plutôt que le contenu de vos réponses, est-ce la façon dont vous envisagez le problème qui intéresse l'auditoire.

Sylvère Monod, vous avez inauguré votre carrière shakespearienne en traduisant une pièce fortement rhétorique et, de surcroît, patriotique et guerrière. N'était-ce pas une tentation de pratiquer l'archaïsme, ne serait-ce que pour rendre sensible la distance qui nous sépare de cette pièce aux accents épiques?

Sylvère MONOD. Au temps de mon inexpérience totale, j'ai cru à l'opportunité d'un effort pour rendre le français de ma 
traduction d'Henry $V$ plus contemporain de Shakespeare que celui que j'utilisais dans la vie courante et dans la plupart de mes travaux littéraires. Je me souviens que je m'étais soumis à un exercice délectable : la lecture ou la relecture des Essais de Montaigne. J'y trouvais des tournures que je croyais pouvoir utiliser; un seul exemple me vient à l'esprit : Montaigne écrit à propos d'un certain sujet «j'en avais fait du tout mon étude»; ce «du tout» signifie «complètement, en totalité»; j'ai glissé cette expression dans ma traduction; Pierre Leyris m'a facilement dissuadé de telles pratiques, qui aboutiraient à une langue hybride, zébrée de disparates. J'y ai donc renoncé de bon cœur. Mon actuel directeur de conscience (traduisante) n'encourage absolument pas la recherche de la patine. Aujourd'hui, je suis convaincu que conférer la vétusté à une langue pour la rapprocher de l'âge du texte qu'on traduit est un exercice prodigieusement artificiel et «du tout» inacceptable. Je conçois qu'on ne partage pas cette opinion, mais elle est désormais fermement ancrée dans mon esprit. Un avantage de la traduction, presque une supériorité par rapport au travail d'un éditeur du texte original, est de rapprocher Shakespeare du lecteur et surtout du spectateur d'aujourd'hui. Le contemporanéiser est une bonne chose et vaut mieux qu'un effort pour se déguiser en son contemporain.

Jean-Pierre MAQUERLOT. On devine, à lire vos traductions, Jean-Pierre Richard, que vous êtes résolument contre l'archaïsme.

Jean-Pierre RICHARD. Je ne conteste à personne le droit de traduire Shakespeare en français archaïsant, si quelqu'un y trouve son plaisir... Bien mené, l'exercice peut être une curiosité enrichissante. Mais, sur ce point, ma priorité est de traduire un rapport d'auteur à spectateurs. Je me pose d'emblée la question suivante: quel rapport linguistique unissait Shakespeare à ses auditoires ? S'adressait-il au public dans une langue marquée à l'époque comme archaïque? Nullement. Je m'efforce donc d'instaurer entre ma traduction et ceux qui l'entendent une relation au français exempte d'archaïsme. Ce qui ne dispense pas, bien entendu, de vouloir traduire au plus serré la préciosité de Roméo, l'emphase de Léonato, la verdeur d'Aaron, la sensualité de Tamora, le lyrisme d'Othello, l'incohérence de Léontès ou toute autre nuance qui vient colorer à l'occasion le discours de tel ou tel personnage.

Jean-Pierre MAQUERLOT. Si je vous suis, c'est par fidélité à l'esprit et à la lettre du théâtre shakespearien que vous refusez l'archaïsme? 
Jean-Pierre RICHARD. Le cas de Shakespeare me rappelle celui de Molière. Que répond l'Administrateur du Français à ces abonnés qui lui contestent le droit de puiser dans le théâtre contemporain, au motif que la Comédie-Française est née en 1680, doit rester la maison de Molière et jouer les seules pièces inscrites au répertoire classique? S'il est indéniable que le Français date de 1680, Jean-Pierre Miquel rappelle qu'il a été créé par la volonté du roi afin de jouer le théâtre contemporain (de Louis XIV); et il n'existe donc pas aujourd'hui de meilleure façon de rester fidèle à Molière et à l'esprit du Français que d'y accueillir le théâtre de l'an 2000 !

De la même façon, la fidélité historique à la langue de Shakespeare est celle qui admet, avec Charles Sorel, que «c'est le privilège de la traduction de pouvoir être réitérée dans tous les siècles, pour refaire les livres selon la mode qui court» (1664). Parce que Shakespeare écrivait dans la langue de son époque, en traduction il sera toujours moderne. On peut aller jusqu'à se demander si, à cet égard, la traduction n'est pas le seul lieu possible de fidélité totale à Shakespeare, puisque, dans sa propre langue, il a le malheur de vieillir. Shakespeare ne demeure contemporain de luimême qu'en traduction. Et si nous pouvons l'aider à rester jeune...

Jean-Pierre MAQUERLOT. J'ai le sentiment que Jean Malaplate serait quelque peu moins catégorique sur cette question de l'historicité de la langue traductrice.

Jean MALAPLATE. L'historicité en matière de traduction obéit, je pense, à des critères de goût et de mesure. Les traductions actuelles doivent être écrites en langage raisonnablement actuel, mais sans oublier l'époque du texte. Il serait assez vain de pasticher à toute occasion le style et d'emprunter le vocabulaire du temps, mais il serait dommage aussi d'éviter systématiquement des expressions anciennes et savoureuses lorsqu'elles viennent sans effort sous la plume, qu'elles peuvent plaire à un lecteur délicat et cultivé sans poser de problème insurmontable à la plupart des autres. Le texte est de toute façon daté par son contenu intrinsèque, par son rythme, par sa forme générale, ses mœurs, ses personnages. Moderniser à outrance la langue serait introduire dans cet ensemble une disparate injustifiée (et se condamner sans doute aussi à vieillir d'autant plus vite). En sens inverse, la traduction de Dante par Pézard en vieux français est un travail étonnant, mais une distraction d'érudit qui ne rend guère service à un lecteur de notre siècle. 
Jean-Pierre MAQUERLOT. À Jean-Michel Déprats dont les traductions sont sans cesse confrontées à la pratique théâtrale contemporaine, $j$ 'ai envie de poser cette question : doit-on, peut-on «contemporanéiser» Shakespeare, pour reprendre le néologisme de S. Monod ? N'y a-t-il pas certaines limites à ne pas franchir ?

Jean-Michel DÉPRATS. Jean-Pierre Richard a cité Charles Sorel. J'aime également m'y référer. Depuis les premières traductions de La Place et de Le Tourneur, traduire ou retraduire Shakespeare a toujours voulu dire traduire dans la langue du moment, à partir d'un certain ancrage dans la langue et la littérature de la culture traduisante. Yves Bonnefoy met en garde contre la tentation de la traduction archaïsante : «Rien n'est plus dangereux que de rêver de traduire Shakespeare dans une imitation de notre propre langue de la fin du XVIe siècle». Personne d'ailleurs ne s'y est véritablement aventuré. La traduction d'Hamlet par Eugène Morand et Marcel Schwob (1899), qui se veut archaïsante, ne l'est que par intermittences et de façon très atténuée. Michel Vittoz a écrit pour Daniel Mesguich en 1977 un Hamlet aux archaïsmes controuvés, métissé d'un jeu verbal très contemporain, à mi-distance de Ronsard et de Mallarmé, de Maurice Scève et de Lacan. Pierre Leyris (Macbeth, Richard III, Cymbeline...) écrit surtout dans une langue très littéraire, recourant occasionnellement à des calques d'allure archaïsante. Le problème de la traduction archaïsante est qu'elle n'a pas d'horizon. L'écriture dramatique de Robert Garnier ne nous donne aucune idée, même approximative, de l'inventivité, de l'audace syntaxique de la langue shakespearienne. Pourrait-on s'inspirer d'Agrippa d'Aubigné, de Rabelais ? On risque de ne produire qu'un artefact sans authenticité linguistico-littéraire, de fabriquer un «meuble de style», à seule fin de produire un effet d'ancienneté. Mais une actualisation outrancière, la recherche d'une crudité ostentatoire, d'une langue argotique, qui caractérisent des adaptations récentes dans lesquelles la volonté de rapprochement se monnaie en actualisation boulevardière me paraissent encore moins désirables et relèvent d'une démagogie mensongère.

Personnellement, il m'arrive très exceptionnellement de recourir à de discrets archaïsmes syntaxiques (ainsi dans Richard III ces vers de Lady Ann : «Déposez, déposez votre fardeau d'honneur [...]» / Cependant qu'endeuillée je lamente / La chute prématurée du vertueux Lancastre»: «lamenter» employé transitivement au sens de déplorer n'existe qu'en vieux français), mais dans l'ensemble mes traductions sont écrites dans une langue résolument moderne. 


\section{Traduire Shakespeare pour le théâtre d'aujourd'hui : cela implique-t-il forcément une déperdition du sens ou de la littérarité ?}

Jean-Pierre Richard, à observer votre réaction, je soupçonne ai-je tort? - que cette manière de poser le problème ne vous satisfait pas.

Jean-Pierre RICHARD. Comment un rendu exigeant de la théâtralité du texte shakespearien pourrait-il nuire au «sens»? Cela me paraît inconcevable, à moins de raisonner en termes dualistes. Dans cette perspective, à mon avis fautive, l'œuvre aurait, d'un côté, une dimension dramatique et, de l'autre, une réalité littéraire où s'épuiserait son «sens». De ce point de vue, une traduction pour la scène ne livrerait qu'une partie de l'œuvre, une version tronquée, amputée de la chair littéraire, autrement dit du «sens» du texte.

Mais qu'appelle-t-on le «sens» d'un texte littéraire ? Pour ma part, je préfère donner du «sens» une définition dynamique. Qu'elle appartienne à un roman, à un poème ou à une pièce de théâtre, chaque phrase littéraire ne fonctionne-t-elle pas comme une scène de théâtre où se produit le «sens»? «Dans quel sens? Dans quel sens ?» demande sans cesse Alice. En haut? En bas? Devant? Derrière ? À droite? À gauche? De biais? Tout droit ? Etc. En littérature, le sens est avant tout directionnel. Il correspond à la résultante des rapports de force qui se nouent dans une phrase, brève ou longue, simple ou complexe, à mesure que je la découvre, à l'œil ou à l'oreille, ou au bout de mes doigts si je suis aveugle. Si «sens» et «littérarité» coïncident, ils relèvent d'une physique de la langue, d'une énergétique. La phrase existe-t-elle seulement avant que je la mette en branle, que je fasse jouer par ma lecture ou par ma diction les forces et les tensions qui la constituent, les équilibres et les déséquilibres qui seuls en définiront, c'est-à-dire en produiront au bout du compte le «sens»?

La traduction d'une œuvre littéraire, à quelque genre qu'elle appartienne, me semble ne pouvoir être que d'ordre dramatique, la traduction d'un texte théâtral représentant le cas de figure le moins problématique, dans la mesure où, en l'occurrence, le procédé constitutif du sens s'affiche ouvertement comme étant d'ordre dramatique. Je ne vois donc pas comment traduire une pièce de théâtre pourrait nous priver d'une partie du «sens» de l'œuvre traduite, puisque le théâtre est justement le lieu où le théâtre du sens s'affiche, où le jeu de forces constitutif du sens prend vie, se fait répliques, jeux de scène et personnages... 


\section{Jean-Pierre MAQUERLOT. Sur un exemple, cela donne quoi ?}

Jean-Pierre RICHARD. Une fois traduit en français le discours de Titus Andronicus aux pierres, d'où proviendrait le sentiment que le «sens» de la scène en aurait pâti ? On aura pris soin de faire jouer dans la traduction ces deux forces opposées qui traversent le personnage et le déchirent; d'une part sa rhétorique ampoulée au début de la scène, quand chaque vers de cet ancien héros guerrier aboutit à une forme verbale, tend vers l'action :

For pity of mine age, whose youth was spent In dangerous wars whilst you securely slept; For all my blood in Rome's great quarrel shed For all the frosty nights that I have watched [...]

Par pitié pour mon âge, moi dont jeunesse se passa En guerres périlleuses quand vous paisiblement dormiez, Pour tout le sang que le glorieux combat de Rome m'a fait verser,

Pour toutes les nuits de givre où j'ai veillé $[\ldots]$

(Titus Andronicus, III.1.2-5)

et, d'autre part, la relation exclusive qui, notamment à travers l'équilibre des pluriels nominaux («sorrows» / «stones») et le parallélisme des pronoms sujets («I»/ «they»), finit par s'instaurer entre l'homme et la pierre, dans la simplicité radicale du dépouillement :

Therefore I tell my sorrows to the stones [...]

When I do weep, they humbly at my feet

Receive my tears and seem to weep with me.

C'est pourquoi je raconte mes peines à ces pierres [...]

Quand je pleure, elles, humblement à mes pieds,

Recueillent mes larmes et semblent pleurer avec moi.

(Titus Andronicus, III.1.37 ; 41-42)

Malgré l'allitération («peines» / «pierres»), la déperdition de «sens», notamment sur le plan du signifiant phonique, est indéniable. Mais est-elle aggravée parce que c'est du théâtre qu'on traduit ? Au contraire, me semble-t-il. Le caractère dramatique des 
formulations accentue les rapports de force entre les éléments constitutifs du «sens», met en valeur la littérarité du texte. Les tensions sont plus vives, les effets mieux repérables. La traduction du sens, loin d'en souffrir, ne s'en trouve-t-elle pas facilitée ?

Jean-Pierre MAQUERLOT. Ainsi une «bonne» traduction devrait être potentiellement jouable, tout en procurant du plaisir au lecteur. Seriez-vous d'accord avec ce point de vue, Jean Malaplate?

Jean MALAPLATE. Bien sûr, Shakespeare est destiné à être joué autant qu'à être lu, mais l'un n'exclut pas l'autre. Lui-même a conçu son texte dans cet esprit et en le traduisant simplement, on aboutit presque sans y penser à un texte théâtral. Il suffit de se mettre dans la peau du personnage et de l'acteur qui le jouera pour ne pas se laisser entraîner vers un style trop «écrit» ou trop cahotant. Je n'omets jamais pour ma part de me dire le texte français, parallèlement au texte anglais, le cas échéant à haute voix, pour m'assurer qu'il est «bien en bouche» et n'imposera pas de contorsions démesurées (intellectuelles ou vocales) à l'acteur. Du reste, la «littérarité» d'une pièce de théâtre - c'est du moins le cas avec Shakespeare - n'est-elle pas presque tout entière dans sa «théâtralité»?

Jean-Pierre MAQUERL OT. Tout à l'heure, Jean-Michel Déprats disait de Pierre Leyris qu'il traduisait dans une langue très littéraire, ce qui n'était d'ailleurs pas péjoratif mais un simple constat. Et vous-même, Sylvère Monod, diriez-vous que votre Henry $V$ était trop littéraire?

Sylvère MONOD. L'objectif principal de la traduction d'une pièce de Shakespeare est-il la scène ou la bibliothèque, le théâtre ou la lecture ? Je constate que le choix des pièces que j'ai souhaité traduire a été dicté par le cinéma pour Henry $V$ (l'interprétation de Laurence Olivier au lendemain de la guerre nous avait éblouis), et pour Much Ado (dont le film de Kenneth Branagh mettait en vive lumière le charme, le brio, la gaieté) et un peu par le théâtre pour Measure for Measure (souvenirs d'une représentation par l'OUDS) ; je n'oublie pas que j'ai beaucoup joué quand j'étais étudiant et jeune professeur (pas trop mal, me semble-t-il; je suis d'ailleurs convaincu qu'il faut être acteur pour être un conférencier ou un prof efficace); néanmoins je ne suis pas plus homme de théâtre ou expert en théâtre qu'expert en savoir shakespearien. Je peux me reprocher de n'avoir pas été autant que je l'aurais dû spectateur de théâtre shakespearien, mais j'ai des souvenirs merveilleux : un 
Midsummer Night's Dream en plein air à Regent's Park en été 1939 ; un Merchant of Venice à Leeds avec Wilson Knight en Shylock; en France un Charles Dullin en Richard III et une Marguerite Jamois en Lady Macbeth; plusieurs Hamlet, dont Jean-Louis Barrault. Pourtant, quand deux hommes de théâtre, Jo Tréhard, patron du TMC de Caen et mon jeune frère Richard Monod, enseignant en études théâtrales, tous deux prématurément disparus, se sont intéressés à mon Henry $V$, ils n'ont pas jugé ma traduction suffisamment théâtrale; j'ai accepté ce verdict. Une surprise toutefois en novembre 1999: une lettre de la SACEM m'annonce qu'une dame souhaite utiliser ma traduction d'Henry $V$ pour un ballet qu'elle va monter; je ne sais pas quel rôle le texte peut jouer dans une chorégraphie (art qui m'est plus étranger qu'aucun autre), mais j'étais heureux de découvrir que, faute d'être jouable, ma traduction était peut-être dansable! Hélas, hélas, hélas ! avant-hier nouvelle lettre de la SACEM ; l'aimable et estimable dame a renoncé à son projet. Je ne dis pas qu'elle ait eu tort.

Encore une question : je ne sais pas si le vers (du traducteur) est plus, ou moins, jouable que sa prose. Quoi qu'il en soit, il semble évident que traduire une pièce de théâtre - et de Shakespeare ! - en aboutissant à un texte non jouable est un échec cuisant. Un peu moins cuisant peut-être dans une édition bilingue, où je crois que la traduction est moins autonome, car le service que la traduction essaie de rendre au texte n'est pas tout à fait le même que dans les éditions unilingues françaises. En bilingue, la traduction ne se substitue pas au texte, elle peut être là pour faciliter l'accès à l'original, dont nul ne contestera qu'il est jouable.

Jean-Pierre MAQUERLOT. Jean-Pierre Richard parlait d'une «dynamique» du sens que la traduction se doit de restituer, mais cette «dynamique» $n$ 'induit-elle pas - ou n'est-elle pas induite par - une dynamique du corps de l'acteur en train de jouer son texte ? Jean-Michel Déprats, qu'en pensez-vous?

Jean-Michel DÉPRATS. Le concept de théâtralité est, on le sait, polysémique et insaisissable. Surtout quand il s'agit d'évoquer la thêâtralité de la langue. Si chacun convient aujourd'hui que la destination scénique du texte shakespearien est à prendre en compte dans la traduction, il n'est pas sûr qu'on puisse s'entendre sur ce qui est «jouable» et ce qui ne l'est pas, sur les différences entre un texte à lire et un texte à dire, sur la spécificité de la traduction théâtrale. Le rythme dans un texte romanesque est aussi important que dans un texte de théâtre. Pour tenter de cerner l'interaction de la langue et 
du vécu corporel, Brecht parle de "gestus», de langue gestuelle et indique que l'énoncé : «Si ton œil est pour toi un objet de scandale, arrache-le» est un énoncé plus «gestuel», plus théâtral que l'énoncé : «Arrache l'œil qui est pour toi un objet de scandale». Le gestus renseigne sur l'attitude physique du locuteur, sur son comportement vis-à-vis de son partenaire et sur son attitude mentale vis-à-vis de son discours et de sa situation théâtrale. Toutes les propriétés sensibles du vers, son phrasé, son rythme, ses ruptures syntaxiques, sa texture auditive, ses effets allitératifs et assonantiques, ses redondances et ses répétitions, etc. font partie du gestus et contribuent à définir le matériau de jeu de l'acteur, l'inscription virtuelle du geste dans la physique de la langue. La traduction littérale de la fin de la tirade de Richard II sur la mort des rois : «ainsi assujetti / Comment pouvez me dire que je suis roi ?» est plus gestuelle et plus précise pour le jeu du comédien que la traduction oratoire mais peu théâtrale qui globalise deux gestus : «Qu'avez-vous à me dire, esclave comme je le suis, que je suis roi ?» (Pierre Leyris). Mais il n'y a pas lieu d'être trop dogmatique et de concevoir les notions d'oralité et de gestualité de façon trop mécaniste et trop restrictive. Traduire du théâtre ne veut en tout cas pas dire adapter (à l'acteur, à l'époque, aux intentions de la mise en scène...) ni simplifier (morceler les périodes pour rapprocher du style parlé ; élaguer les métaphores par souci de l'immédiateté et de la clarté).

Quand on traduit Shakespeare, le souci de la théâtralité conduit souvent à opter pour une forme de littéralité. L'exactitude du sens n'est pas forcément mieux préservée dans une traduction littéraire ou universitaire. Mais comme pour la question du vers ou de l'historicité de la langue, tout est affaire de souplesse et de pesée individuelle. Il n'est pas deux pièces, deux tirades qui présentent les mêmes caractéristiques et les mêmes difficultés. Par ses propositions concrètes, le traducteur décide à tout moment ce qu'il faut abandonner, ce qu'il faut privilégier (le sens, le rythme, la rime, la syntaxe...). La hiérarchie des priorités dépend de l'interprétation (au sens musical autant qu'herméneutique). Le souci du théâtre est essentiel mais ne répond pas à tout.

Jean-Pierre MAQUERLOT. Le moment est venu de clore ce débat. Nous n'avons pas épuisé le sujet mais, grâce à vous, nous cernons mieux certains enjeux majeurs de la traduction et nous voyons se dessiner plus clairement les grands axes de la problématique à laquelle vous confronte votre pratique de 
traducteurs shakespeariens. Soyez-en vivement remerciés; et puissions-nous avoir d'autres occasions pour reprendre cette discussion fructueuse.

Table ronde retranscrite par Jean-Pierre MAQUERLOT Université de Rouen 